\title{
Correction to: Elastic caching solutions for content dissemination services of ip-based internet technologies prospective
}

\author{
Yahui Meng ${ }^{1}$ - Muhammad Ali Naeem ${ }^{1}$ - Muhammad Sohail ${ }^{2}$. Ali Kashif Bashir ${ }^{3}$. \\ Rashid Ali $^{4} \cdot$ Yousaf Bin Zikria ${ }^{5}$
}

Published online: 3 October 2020

(C) Springer Science+Business Media, LLC, part of Springer Nature 2020

\section{Correction to: Multimedia Tools and Applications (2020) https://doi.org/10.1007/s11042-020-09626-7}

The article title in the original publication of this article contains a mistake. The original article has been corrected.

Publisher's note Springer Nature remains neutral with regard to jurisdictional claims in published maps and institutional affiliations.

The online version of the original article can be found at https://doi.org/10.1007/s11042-020-09626-7

Rashid Ali

rashidali@sejong.ac.kr

Yousaf Bin Zikria

yousafbinzikria@ynu.ac.kr

Yahui Meng

mengyahui@gdupt.edu.cn

Muhammad Ali Naeem

malinaeem7@gmail.com

Muhammad Sohail

engrsohailaslam@gmail.com

Ali Kashif Bashir

a.bashir@mmu.ac.uk

1 School of Science, Guangdong University of Petrochemical Technology, Maoming 525000, China

2 School of Computer Science and Communication Engineering, Jiangsu University, Zhenjiang 212013 Jiangsu, People’s Republic of China

3 Department of Computing and Mathematics, Manchester Metropolitan University, Manchester, UK

4 School of Intelligent Mechatronics Engineering, Sejong University, Seoul 05006, Republic of Korea

5 Department of Information and Communication Engineering, Yeungnam University, Gyeongsan 38541, Republic of Korea 